\title{
Probing MACHOs in M31
}

\author{
Jelte De Jong* \\ Kapteyn Astron. Inst., Groningen, The Netherlands \\ E-mail: jdejongeastro.rug.nl
}

\section{and the MEGA team ${ }^{\dagger}$}

From observations, little is known about the nature and structure of galaxy halos. Stellar streams have been found around the Galaxy and our nearest neighbour, M31, presumably remnants of past accretion events. These represent only a very small fraction of the total mass of the halos. From microlensing surveys towards the Magellanic Clouds there are indications that part of the Galactic halo might consist of dark compact objects, but the exact amount is uncertain. M31 provides a significantly better target for a microlensing survey than the Magellanic Clouds. Due to the lensing geometry and the availability of lines-of-sight through very dense parts of the M31 halo, the microlensing rates are greatly enhanced. The Microlensing Exploration of the Galaxy and Andromeda (MEGA) is performing a large-scale microlensing survey towards M31 using several telescopes. We present the first candidate microlensing events from the INT data (de Jong et al. 2004). These results are preliminary, but suggestive of the presence of a microlensing halo.

Baryons in Dark Matter Halos

5-9 October 2004

Novigrad, Croatia

\footnotetext{
${ }^{*}$ Speaker.

${ }^{\dagger}$ the MEGA team: A. P. S. Crotts, K. H. Kuijken, P. D. Sackett, W. J. Sutherland, R. R. Uglesich, E. A. Baltz, P. Cseresnjes, G. Gyuk, L. M. Widrow
} 


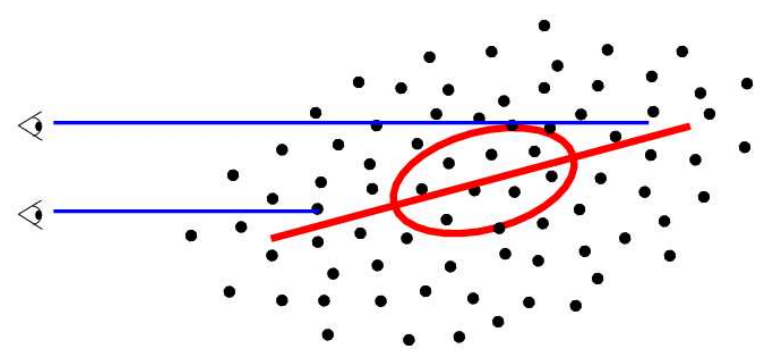

Figure 1: M31's disk has in inclination of 77 degrees to our line-of-sight. Because of this, the path length through the dark halo is much lnger towards the far side of the disk, greatly increasing the microlensing probability with respect to the near side. Therefore, the microlensing signature of the halo will be much more asymmetric than that of microlensing by stars in M31.

\section{Introduction}

If the dark halo of our galaxy (partly) consists of massive compact objects (MACHOs), then they should reveal their presence by magnifying background sources when passing exactly in front of them, as first suggested by Paczynski [1]. Microlensing surveys in the direction of the Magellanic Clouds have shown that at most $20 \%$ of the halo mass might be in compact objects of $\sim 0.5 M_{\odot}[2,3]$. However, the uncertainty is still large, because both the nature and the location of the lenses is not clear.

M31 provides a significantly better target for a microlensing survey than the Magellanic Clouds [4]. A favourable lensing geometry, very high surface density of background stars and the availability of lines-of-sight through dense parts of the M31 halo combine to give microlensing rate up to an order of magnitude higher than for the Magellanic Clouds. Furthermore, due to the high inclination of M31, the microlensing optical depth due to a possible MACHO halo varies strongly across the face of the galaxy (figure 1).

\section{The MEGA project}

The Microlensing Exploration of the Galaxy and Andromeda (MEGA) is performing a multiyear, multi-telescope microlensing survey towards M31. MEGA is performing an intensive observing program, using several telescopes with wide field imaging capabilities. Telescopes that are used include the Isaac Newton Telescope (INT) on La Palma, the $1.3 \mathrm{~m}$ and $2.4 \mathrm{~m}$ telescopes of the MDM Observatory and the KPNO 4m telescope. For the layout of the INT fields in M31, see figure 4. Data is also obtained with the Hubble Space Telescope to identify the individual source stars of microlensing events.

\section{Our method}

Because of the high stellar density in M31 and its large distance, the background source stars are usually only resolved while being lensed and sufficiently magnified. We use difference image photometry [5] to detect and photometer variable and often unresolved sources in M31. 

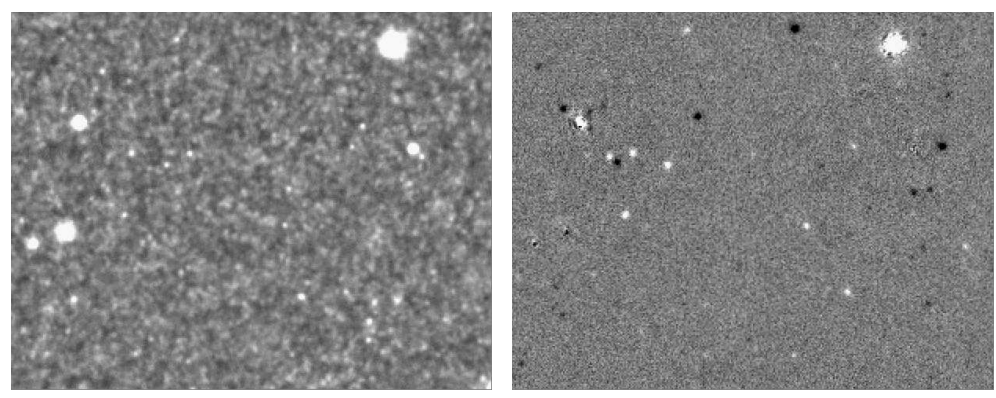

Figure 2: Left: Small part of a single night image obtained with the Isaac Newton Telescope. Only the brightest stars are resolved, each pixel contains flux from several stars. Right: After subtracting a high signal-to-noise reference image, this is the resulting difference image. Variable sources show up as positive or negative residuals on a background dominated by shot-noise. Note that bright stars also leave some residuals due to imperfect subtraction.

The standard data reduction for the INT Wide Field Camera (WFC) images is performed in IRAF. A high signal-to-noise reference image is then created from some of the best seeing frames taken over a whole observing season (Aug-Jan). Nightly images are subtracted from this reference image, resulting in difference images in which variable sources remain as positive or negative residuals. Before subtracting two images the point spread functions (PSFs) are matched by degrading the best seeing frame (usually the reference frame) to the same seeing as the worst seeing frame. The variable sources can be detected easily in the difference images, after which aperture photometry is used to construct lightcurves. An example of the image subtraction method is shown in figure 2 .

\section{First microlensing candidates}

Based on an analysis of the first two years of data obtained at the Isaac Newton Telescope on La Palma we find 14 candidate microlensing events [6], 12 of which are new and 2 have been reported previously by the POINT-AGAPE group [7]. In figure 3 we show our lightcurves of event MEGA-ML-7, the highest signal-to-noise event in the sample. Figure 4 shows the positions of the 14 candidate events in M31. A comparison of their spatial distribution with that of long-period variable stars shows that the events are distributed more asymmetrically than the variables, with

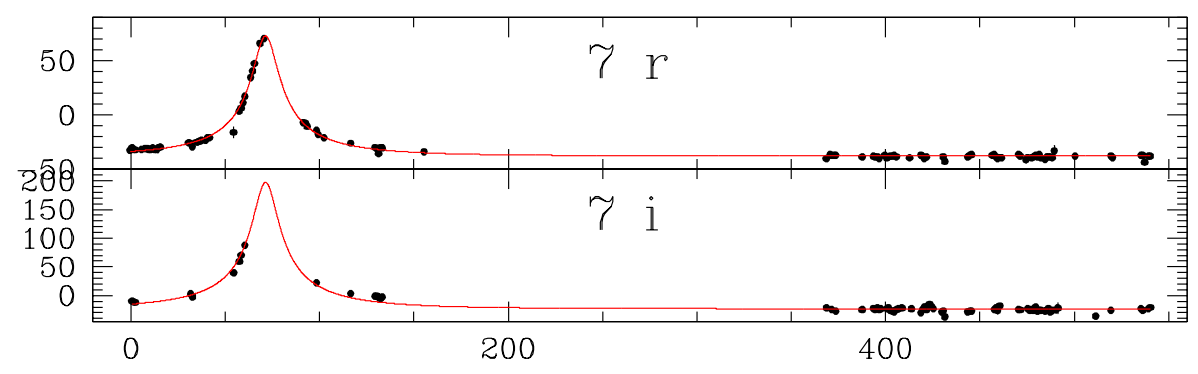

Figure 3: Lightcurves in r' and i' of the candidate microlensing event MEGA-ML-7. This is the highest signal-to-noise event in the sample, with a peak difference flux corresponding to 19.3 magnitudes in r'. 


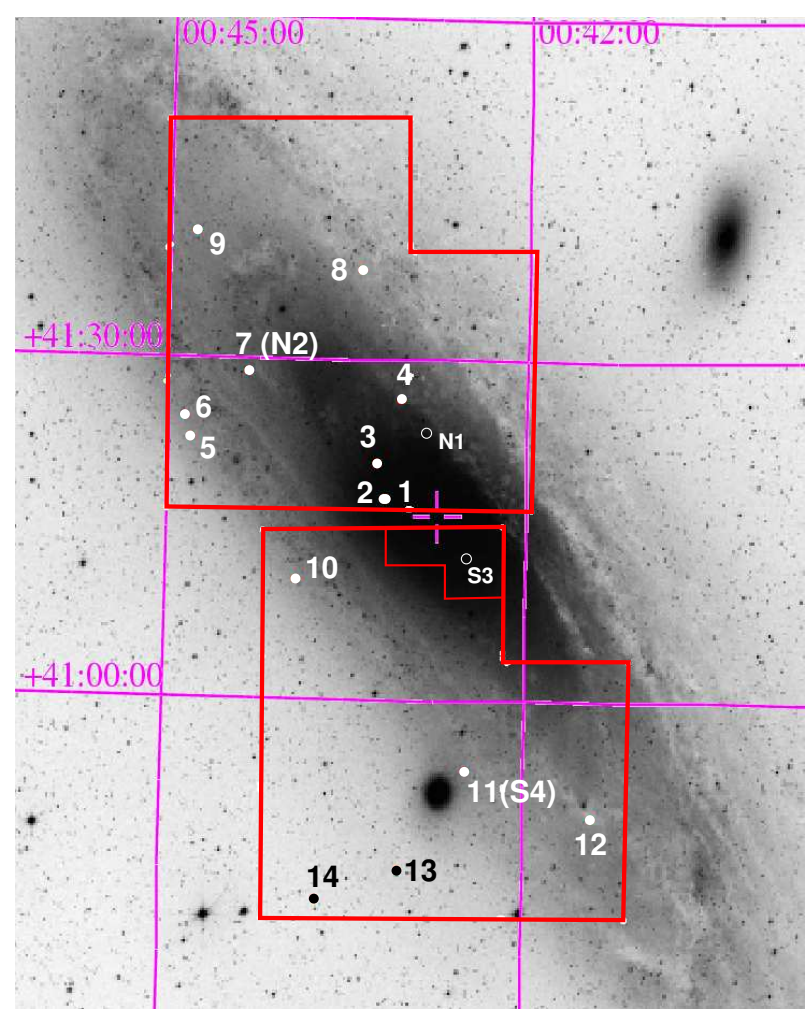

Figure 4: Layout of the INT fields in M31 and positions of the candidate microlensing events. The 14 candidate events are indicated by the numbers 1 through 14 . Events 7 and 11 correspond to events $\mathrm{N} 2$ and $\mathrm{S} 4$ reported by [7]. The locations of their events $\mathrm{N} 1$ and $\mathrm{S} 3$ are indicated by open circles.

more events on the far side. Although the current analysis is preliminary and the asymmetry nog highly significant, this result is suggestive of microlensing by th M31 halo.

\section{References}

[1] Paczynski, B., 1986, ApJ 304, 1

[2] Alcock, C. et al. 2000, ApJ 542, 281

[3] Lasserre, T. et al. 2000, A\&A 355, L39

[4] Crotss, A. P. S., 1992, ApJL 399, L43

[5] Tomaney, A. B. \& Crotts, A. P. S., 1996, AJ 11122872

[6] de Jong, J. T. .A. et al. 2004, A\&A 417, 461

[7] Paulin-Henriksson, S. et al. 2003, A\&A 405, 15 